ELECTRONIC RESEARCH ANNOUNCEMENTS OF THE AMERICAN MATHEMATICAL SOCIETY

Volume 6, Pages 50-51 (July 19, 2000)

S $1079-6762(00) 00080-9$

\title{
ON NON-SPECHTIANNESS OF THE VARIETY OF ASSOCIATIVE RINGS THAT SATISFY THE IDENTITY $x^{32}=0$
}

\author{
A. V. GRISHIN
}

(Communicated by Efim Zelmanov)

Abstract. In this paper we construct examples of $T$-spaces and $T$-ideals over a field of characteristic 2 , which do not have the finite basis property.

Since the end of the sixties and up to date a number of examples of algebraic objects without the finite basis property have been constructed (for groups, Lie algebras, and certain other rings close to associative). On the other hand, for the associative rings and several other classes of rings a number of positive results have been obtained. For finite rings it is the theorem of Lvov and Kruse 1, 2, for algebras over a field of characteristic 0 it is Kemer's theorem (see [3]), and for $T$-spaces over a field of characteristic 0 it is the author's result (see [4]).

At some stage a majority of mathematicians working in $P I$-rings believed that any associative algebra over a sufficiently "good" ring (for example, over a field or the integers) has the finite basis property. Nevertheless, in this paper we show that this is not true.

Definition. Let $k$ be an associative and commutative ring with unity, and let $F=k\left\langle x_{1}, \ldots, x_{i}, \ldots\right\rangle$ be a countably generated associative free algebra over $k$. Any $k$-submodule $V$ of $F$, closed under substitutions that replace $x_{i}$ by an arbitrary element of $F$, is said to be a $T$-module of $F$.

In particular, if $k$ is a field, then such a $T$-module is called a $T$-space; if $k=\mathbb{Z}$, then it is called a $T$-group. If a $T$-module is also an ideal of $F$, then it is called a T-ideal.

We show that in a free $\mathbb{Z}$-algebra $\mathbb{Z}\left\langle x_{1}, \ldots, x_{i}, \ldots\right\rangle$ there exist infinite ascending chains of $T$-ideals. Also it will be shown that in the relatively free algebra $\mathbb{Z}\left\langle x_{1}, \ldots, x_{i}, \ldots\right\rangle /\left(\left[x_{1},\left[x_{2}, x_{3}\right]\right]\right)^{T}$ there exist infinite ascending chains of $T$-groups. We present explicit constructions.

Theorem 1. Let $k$ be a field of characteristic 2, and let $\Omega$ be the $T$-ideal of $F$ generated by the polynomial $\left[x_{1},\left[x_{2}, x_{3}\right]\right]$. Then the system of polynomials $x_{1}^{2} \cdots x_{n}^{2}$, $n \in \mathbb{N}$, generates a $T$-space of the relatively free algebra $F / \Omega$, which does not have the finite basis property.

We say that a variety $\mathfrak{M}$ of rings has the Specht property if any subvariety of $\mathfrak{M}$ has the finite basis property.

Received by the editors March 22, 1999.

2000 Mathematics Subject Classification. Primary 16R10. 
The following statement can be deduced from Theorem 1 (but the proof is not trivial).

Theorem 2. Let $k$ be a field of characteristic 2 . Then the $T$-ideal generated in the free algebra $F=k\left\langle x_{1}, \ldots, x_{i}, \ldots, y_{1}, y_{2}, z_{1}, z_{2}\right\rangle$ by the polynomials

$$
\begin{gathered}
g_{0}=y_{1}^{4} z_{1}^{4} z_{2}^{4} y_{2}^{4} y_{1}^{4} z_{1}^{4} z_{2}^{4} y_{2}^{4}, \\
g_{n}=y_{1}^{4} z_{1}^{4} x_{1}^{2} \cdots x_{n}^{2} z_{2}^{4} y_{2}^{4} y_{1}^{4} z_{1}^{4} x_{n+1}^{2} \cdots x_{2 n}^{2} z_{2}^{4} y_{2}^{4},
\end{gathered}
$$

where $n \in \mathbb{N}$, does not have the finite basis property.

Corollary 1. Let $A$ be a commutative ring which can be mapped homomorphically onto a $\mathbb{Z}_{2}$-algebra with unity (for example, $A=\mathbb{Z}$ ). Then the polynomials given in Theorems 1 and 2 generate a T-ideal (T-module, T-group) of $A\left\langle x_{1}, \ldots\right.$, $\left.x_{i}, \ldots, y_{1}, y_{2}, z_{1}, z_{2}\right\rangle$, which is not finitely generated.

Corollary 2. The variety of associative rings does not have the Specht property. Furthermore, the variety of associative rings that satisfy the identity $x^{32}=0$ does not have it either.

The details of the proofs of these theorems are given in [5] .

\section{REFERENCES}

[1] I. V. Lvov, Varieties of associative rings. I, Algebra i Logika 12 (1973), 269-297. (Russian) MR 52:10802

[2] B. I. Kruse, Identities satisfied by a finite ring, J. Algebra 26 (1973), 298-318. MR 48:4025

[3] A. R. Kemer, Finite basability of identities of associative algebras, Algebra i Logika 26 (1987), 597-641; English transl., Algebra and Logic 26 (1987), 362-397. MR 90b:08008

[4] A. V. Grishin, On the finite basis property of abstract T-spaces, Fundamental'naya i Prikladnaya Matematika 1 (1995), 669-700. (Russian)

[5] A. V. Grishin, Examples of T-spaces and T-ideals over a field of characteristic 2 without the finite basis property, Fundamental'naya i Prikladnaya Matematika 5 (1999), 101-118. (Russian)

Department of Mathematics, Moscow State Pedagogical University, KrasnoprudNAYA 14, Moscow, Russia

E-mail address: markov@mech.math.msu.su 